\title{
18F-DOPA PET/CT and 68Ga-DOTANOC PET/CT scans as diagnostic tools in focal congenital hyperinsulinism: a blinded evaluation
}

\author{
Charlotte Dahl Christiansen ${ }^{1,2}$ • Henrik Petersen ${ }^{3}$ - Anne Lerberg Nielsen ${ }^{3}$. \\ Sönke Detlefsen ${ }^{2,4}$ • Klaus Brusgaard ${ }^{5}$ - Lars Rasmussen $^{6}$ • Maria Melikyan ${ }^{7}$. \\ Klas Ekström $^{8}$ • Evgenia Globa ${ }^{9}$ - Annett Helleskov Rasmussen ${ }^{1,2}$ • Claus Hovendal ${ }^{6}$. \\ Henrik Thybo Christesen ${ }^{1,2,10,11}$
}

Received: 26 June 2017 / Accepted: 20 October 2017 / Published online: 8 November 2017

(C) The Author(s) 2017. This article is an open access publication

\begin{abstract}
Purpose Focal congenital hyperinsulinism (CHI) is curable by surgery, which is why identification of the focal lesion is crucial. We aimed to determine the use of $18 \mathrm{~F}-$ fluorodihydroxyphenylalanine (18F-DOPA) PET/CT vs. 68Ga1,4,7,10-tetraazacyclododecane-1,4,7,10-tetraacetic-acid-1Nal3-octreotide (68Ga-DOTANOC) PET/CT as diagnostic tools in focal CHI.

Methods PET/CT scans of children with CHI admitted to Odense University Hospital between August 2005 and June 2016 were retrospectively evaluated visually and by their maximal standardized uptake values $\left(\mathrm{SUV}_{\max }\right)$ by two independent examiners, blinded for clinical, surgical and pathological data. Pancreatic histology was used as the gold standard. For patients without surgery, the genetic profile served as the gold standard.
\end{abstract}

Electronic supplementary material The online version of this article (https://doi.org/10.1007/s00259-017-3867-1) contains supplementary material, which is available to authorized users.

Henrik Thybo Christesen henrik.christesen@rsyd.dk

1 Hans Christian Andersen Children's Hospital, Odense University Hospital, Odense, Denmark

2 Department of Clinical Research, University of Southern Denmark, Odense, Denmark

3 Department of Nuclear Medicine, Odense University Hospital, Odense, Denmark

4 Department of Pathology, Odense University Hospital, Odense, Denmark

5 Department of Clinical Genetics, Odense University Hospital, Odense, Denmark
Results Fifty-five CHI patients were examined by PET/CT $(18 \mathrm{~F}-\mathrm{DOPA} n=53,68 \mathrm{Ga}-\mathrm{DOTANOC} n=18)$. Surgery was performed in 34 patients, no surgery in 21 patients. Fifty-one patients had a classifiable outcome, either by histology ( $n=33,22$ focal lesions, 11 non-focal) or by genetics ( $n=18$, all non-focal). The predictive performance of $18 \mathrm{~F}$ DOPA PET/CT to identify focal CHI was identical by visualand cut-off-based evaluation: sensitivity $(95 \% \mathrm{CI})$ of $1(0.85-$ $1)$; specificity of $0.96(0.82-0.99)$. The optimal 18F-DOPA

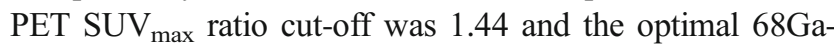
DOTANOC PET SUV max $_{\text {ax }}$ cut-off was $6.77 \mathrm{~g} / \mathrm{ml}$. The area under the receiver operating curve was $0.98(0.93-1)$ for 18F-DOPA PET vs. $0.71(0.43-0.95)$ for 68Ga-DOTANOC PET $(p<0.03)$. In patients subjected to surgery, localization of the focal lesion was correct in $91 \%$, and $100 \%$, by $18 \mathrm{~F}-\mathrm{DOPA}$ $\mathrm{PET} / \mathrm{CT}$ and 68Ga-DOTANOC PET/CT, respectively.

6 Department of Abdominal Surgery, Odense University Hospital, Odense, Denmark

Endocrine Research Centre, Moscow, Russia

8 Astrid Lindgren Children's Hospital, Karolinska Hospital, Stockholm, Sweden

$9 \quad$ Ukrainian Center of Endocrine Surgery, Endocrine Organs and Tissue Transplantation, MOH of Ukraine, Kyiv, Ukraine

10 Odense Pancreas Center (OPAC), Odense University Hospital, Odense, Denmark

11 Department of Paediatrics, Odense University Hospital, Sdr. Blvd. 29, DK-5000 Odense C, Denmark 
Conclusion 18F-DOPA PET/CT was excellent in predicting focal $\mathrm{CHI}$ and superior compared to 68Ga-DOTANOC PET/ CT. Further use of 68GA-DOTANOC PET/CT in predicting focal $\mathrm{CHI}$ is discouraged.

Keywords Congenital hyperinsulinism · Hypoglycaemia · Positron emission tomography $\cdot$ Endocrine pancreas $\cdot$ Genetic diseases

\section{Introduction}

Congenital hyperinsulinism (CHI) is a rare, heterogeneous disease characterized by inappropriate insulin secretion from pancreatic islet $\beta$-cells resulting in hypoglycaemia [1]. The approximate incidence of persistent $\mathrm{CHI}$ is $1 / 40,000$ live births in countries without founder mutations $[2,3]$. The elevated serum insulin results in hypoketotic hypoglycaemia and a clinical presentation ranging from weak symptoms to loss of consciousness and seizures with a high risk of brain damage $[1,4]$.

The two major histological forms of $\mathrm{CHI}$ are the focal and the diffuse forms. Focal CHI occurs in $40-50 \%$ of cases [5] and is defined as a restricted pancreatic area with adenomatous $\beta$-cell hyperplasia, resulting in a lesion composed of confluent islets of Langerhans [6, 7]. Diffuse CHI involves the islets of Langerhans throughout the pancreas and is histologically characterized by hypertrophy of a few $\beta$-cell nuclei in most islets of Langerhans. In $50-66 \%$ of patients with diffuse CHI, mutations are found in the $\mathrm{K}_{\mathrm{ATP}}$-channel genes $A B C C 8$ or $K C N J 11$, coding for the sulphonylurea receptor 1 (SUR1) and the potassium inward rectifier 6.2 (Kir6.2), respectively [6, 8]. Recessive inactivating mutations in $A B C C 8 / K C N J 11$ are the most common causes of medically unresponsive diffuse $\mathrm{CHI}$ [9], whereas dominantly inherited $A B C C 8 / K C N J 11$ mutations typically are medically responsive [1].

Focal CHI is strongly associated with a heterozygous, paternally inherited $\mathrm{K}_{\mathrm{ATP}}$-channel germline mutation, as this combined with a somatic loss of heterozygosity in chromosome $11 \mathrm{p} 15$ in a focal region of the pancreas results in hemizygosity of the paternal $A B C C 8 / K C N J 11$ mutation and loss of maternally expressed tumor suppressors [1].

The primary treatment goal is to increase and maintain blood glucose concentration above at least $3.5 \mathrm{mmol} / \mathrm{L}$ to avoid brain damage [10]. The treatment modalities include diet, i.v. glucose infusion, anti-insulin medical therapy (primarily diazoxide and octreotide) and pancreatic surgery [1]. Focal CHI is curable after a focal enucleation, or partial pancreatectomy, without subsequent risk of diabetes or malabsorption $[9,11]$. Surgery for diffuse CHI should be restricted to medical non-responders and ranges from partial to subtotal pancreatectomy; the first is most often ineffective, and the latter imposes a high risk of diabetes mellitus and exocrine pancreatic insufficiency $[11,12]$.

Today, 18F-fluoro-dihydroxyphenylalanine (18F-DOPA) PET is the preferred tool in discerning focal from non-focal CHI [13]. L-DOPA is converted to dopamine by the aromatic amino acid decarboxylase enzyme in neuroendocrine cells [14]. The combined use of 18F-DOPA PET and CT [15-19] or MRI [20, 21] allows for a precise localization of the focal process prior to surgery. The preoperative identification of a focal lesion can be made by visual $[15,16,18,21]$ or quantitative $[17,19,20,22]$ methods. By the quantitative approach, a high standardized uptake value (SUV) ratio is used to identify the focal lesion. However, published diagnostic SUV ratio cut-offs range from 1.2 [22] to 1.5 [17, 19, 20]. 18F-DOPA is not available in every country and is more difficult to manufacture than another tracer, $68 \mathrm{Ga}-1,4,7,10-$ tetraazacyclododecane-1,4,7,10-tetraacetic acid-1-Nal3octreotide (68Ga-DOTANOC). 68Ga-DOTANOC is a somatostatin analogue radiotracer with high affinity to the somatostatin receptor (SSTR) subtypes 2, 3, and 5. All the SSTR subtypes are variably expressed in endocrine cells of the islets of Langerhans [23], but the diagnostic value of $68 \mathrm{Ga}$ DOTANOC PET/CT in the diagnosis of focal CHI is unknown [24]. As the waiting time to expert treatment is essential for the cerebral prognosis [4], a more easily available alternative to the 18F-DOPA PET would be desirable to shorten time to curative surgery for focal $\mathrm{CHI}$ patients born far from these centers.

We aimed to evaluate 18F-DOPA PET/CT and 68GaDOTANOC PET/CT as diagnostics tools in focal CHI preoperatively, analyzed visually or quantitatively, by ratio of maximal SUV values $\left(\mathrm{SUV}_{\max }\right.$ ratio) for $18 \mathrm{~F}-\mathrm{DOPA} \mathrm{PET} / \mathrm{CT}$, or $\mathrm{SUV}_{\text {max }}$ for 68Ga-DOTANOC PET/CT.

\section{Patients and methods}

We retrospectively investigated children with $\mathrm{CHI}$ admitted to the International Hyperinsulinism Center at Odense University Hospital, Denmark, between August 2005 and June 2016, subjected to 18F-DOPA PET/CT and/or 68GaDOTANOC PET/CT scan.

The CHI diagnosis was based on an inappropriately elevated p-insulin concentration during hypoglycaemia. Patients above 18 years were excluded, leading to final inclusion of 55 patients.

Anti-insulin medication was stopped two days prior to the PET/CT scan, allowing verification of the diagnosis of persistent $\mathrm{CHI}$ and evaluation of disease severity. To minimize stress for the patients and movement artifacts, 18F-DOPA PET/CT scans were obtained under general anesthesia (60min acquisition time), while most 68Ga-DOTANOC PET/ CT scans were performed under sedation (5-min acquisition time). 


\section{PET/CT-based assessment}

PET/CT scans were acquired on a GE Discovery PET/CT scanner (GE Medical System, Waukesha, WI, USA) and analyzed on a Dexus AW server 2.0. 18F-DOPA was produced by the electrophilic method. The patients were injected with $18 \mathrm{~F}-$ DOPA or $68 \mathrm{Ga}-\mathrm{DOTANOC} 4 \mathrm{MBq} / \mathrm{kg}$, minimum $30 \mathrm{MBq}$. One field of view (FOV; acquisition time $5 \mathrm{~min} / \mathrm{FOV}$ ) was obtained over the pancreatic region at 10,30 and $60 \mathrm{~min}$. After injection, $68 \mathrm{Ga}$-DOTANOC scans were obtained at 10 , 30 and $60 \min (n=6)$, or at $30 \min (n=1), 45 \min (n=8)$ or $60 \min (n=3)$. A low-dose CT scan was performed prior to the first scan to be used for attenuation correction; acquisition parameters were $80-100 \mathrm{kV}, 30-40 \mathrm{~mA}$, rotation $0.8 \mathrm{~s}$ and pitch $0.984: 1$. A contrast-enhanced diagnostic CT scan over the pancreatic region was performed afterwards in all but one patient with suspicion of focal $\mathrm{CHI}$ to aid surgeons to localize the focal lesion: acquisition parameters were $120 \mathrm{kV}, 30-400 \mathrm{~mA}$, SmartmA, rotation $0,8 \mathrm{~s}$, pitch $0.984: 1$ and noise index of 10 . The CT scans were iteratively reconstructed to minimize radiation dose. Diagnostic CT scans were evaluated by radiologists.

$\mathrm{PET} / \mathrm{CT}$ scans were anonymized and case numbers of $18 \mathrm{~F}-$ DOPA, and 68Ga-DOTANOC scans were randomized independently from each other. $\mathrm{SUV}_{\max }$ values were measured in $\mathrm{g} / \mathrm{ml}$ and the region of interest (ROI) was shaped as a sphere. Results of the attenuation-corrected 18F-DOPA and 68Ga-DOTANOC $\mathrm{PET} / \mathrm{CT}$ were evaluated by two independent researchers (C.D.C and A.L.N), blinded for all other clinical, radiological, surgical and pathological data. Disagreement was defined as a difference in $\mathrm{SUV}_{\max }>10 \%$, a SUV ratio difference $>0.2$ or a difference in visual or quantitative conclusion; the latter applied for $18 \mathrm{~F}-$ DOPA PET based on a predefined SUV ratio cut-off $=1.45$. Disagreements were resolved by a third party (H.P.). Two patients enrolled in the study were evaluated by A.T and M.H.V (see Acknowledgements) due to blinding issues, and disagreement was resolved by a third party (H.P).

The PET/CT scans were evaluated in the following order: 1) Visual method: a visually higher uptake of radiotracer in a part of the pancreas was considered positive for a focal process, and location was noted. A uniform uptake throughout the pancreas was considered diffuse. 2) Measurement of the $\mathrm{SUV}_{\max }$ ratio in the pancreas: $\mathrm{SUV}_{\max }$ in the part of the pancreas that visually appeared to be abnormal, divided by $\mathrm{SUV}_{\text {max }}$ in the part of the pancreas that visually appeared to be homogenous and normal (body or head). The SUV $\mathrm{Sax}_{\text {max }}$ ratio was measured at 10,30 and $60 \mathrm{~min}$ after injection. The highest $\mathrm{SUV}_{\max }$ ratio was used and location was noted.

Patient files were evaluated after the blinded PET/CT evaluation. The following data were extracted from the medical records; sex, age at disease onset and at admission, country of origin, family history, genetic analyses, medication, maximal intravenous glucose infusion rate (off-medication prior to $\mathrm{PET} / \mathrm{CT}$ scan) and lowest recorded blood glucose concentration. Insulin, proinsulin and C-peptide concentrations were obtained during hypoglycaemia, defined as a blood glucose below $2.5 \mathrm{mmol} / \mathrm{L}$ (neonatal period), or below $3.2 \mathrm{mmol} / \mathrm{L}$ (thereafter). The location and size of the lesions, results of frozen section microscopy and the final histological diagnoses were retrieved from the pathology report.

\section{Histopathological analysis}

The histological diagnoses were retrieved from the files of the Dept. of Pathology, Odense University Hospital, Denmark, in all cases with surgery, except two patients who were operated upon abroad. For the final histological diagnoses, the pathology protocol for formalin-fixed, paraffin-embedded pancreatic tissue included hematoxylin-eosin of 4- $\mu$ m-thick sections; immunohistochemical staining using the BenchMark Ultra immunostainer (Ventana Medical Systems, Tucson, AZ, USA) with the OptiView-DAB detection kit (Ventana Medical Systems, Tucson, AZ, USA); nuclear counterstaining with the BenchMark Ultra instrument using Hematoxylin II (Ventana Medical Systems, Tucson, AZ, USA), and coverslipping using a Tissue-Tek Film coverslipper (Sakura, Alphen aan den Rijn, The Netherlands). In most cases, immunohistochemical examination included synaptophysin, chromogranin A, insulin, glucagon, somatostatin, and the maternally expressed tumor suppressor $\mathrm{p} 57$. For preoperative frozen section analysis, 4- $\mu$ m-thick frozen sections were stained with hematoxylin-eosin and tolouidin blue. Besides, particularly when the tissue specimens submitted for frozen section were small $(<5 \mathrm{~mm})$, manual immunohistochemical staining of frozen sections for synaptophysin and insulin was performed.

\section{Genetic analysis}

Prior to 2007, genetic analyses were performed by denaturing high-pressure liquid chromatography (dHPLC) analysis of $A B C C 8$ and $K C N J 11$ as previously described [25]. A positive dHPLC was followed by Sanger sequencing [26]. From 2007 to 2013, analysis of ABCC8, KCNJ11, GLUD1, GCK, HNF1-alfa and HNF4-alfa was performed using Sanger sequencing [26]; from 2011, also including HNF1-beta, and from 2012, also including $H A D H, M C T 1$ and UCP2. From 2013 and onwards, analysis of the before-mentioned genes was performed using next-generation sequencing (NGS) as previously described [27] followed by Sanger sequencing for confirmation of mutations found by NGS. Pathogenicity analyses were performed using multiple software programs [28-34]. Only previously reported mutations or rare DNA variants predicted by 
software analysis to be disease-causing were accepted as pathogenic.

\section{Statistics}

Continuous variables were expressed by the median and interquartile ranges (IQR); categorical data in number and percentage. To estimate reproducibility, kappa values were calculated for categorical variables and intraclass correlation coefficients (ICC) for numerical variables. Reproducibility tests were made between patients analyzed by C.D.C and A.L.N.

Histopathology after surgery was used as the primary gold standard to discern focal from non-focal (diffuse or atypical) $\mathrm{CHI}$. In patients not subjected to surgery, the absence of a heterozygous, paternal $A B C C 8 / K C N J 11$ mutation was used as a secondary gold standard for non-focal CHI (no patients showed evidence of a dominant, paternal $A B C C 8 / K C N J 11$ mutation). If no gold standard was available, the patient was excluded from analyses of test performance.

The performance of the PET/CT scans was tested on both the combined gold standard outcome (surgical and nonsurgical patients) and on the primary gold standard outcome (histologically confirmed focal lesion after surgery).

To investigate test performance, receiver operating curves (ROC) were drawn from the $\mathrm{SUV}_{\text {max }}$ ratio (18F-DOPA PET) and the $\mathrm{SUV}_{\max }$ (68Ga-DOTANOC PET). The optimal cutoff to maximize accuracy, the area under the curve (AUC) and the corresponding $95 \%$ CI were calculated by a bootstrap of 10,000 .

Sensitivity, specificity, positive and negative predictive values (PPV and NPV, respectively) were calculated for the visual, or SUV-based, diagnostic prediction of focal CHI, including for the SUV ratio cut-off of 1.44 [21]. The 95\% CIs were calculated by the Wilson method [35]. Comparison of $\mathrm{SUV}_{\max }$ ratios and $\mathrm{SUV}_{\max }$ values between groups was done using Mann-Whitney $\mathrm{U}$ tests. Comparison of PET/CT scan test performances was done by their 95\% CI [36]. Comparison of superiority of ROC AUC of 18F-DOPA PET over 68GaDOTANOC PET was done using a non-paired bootstrap of 10,000 .

Disease severity expressed as lowest blood glucose, or maximal glucose infusion demand without medication prior to $\mathrm{PET} / \mathrm{CT}$ scan, was compared to the $\mathrm{SUV}_{\max }$ ratio of $18 \mathrm{~F}-$ DOPA PET in focal CHI by a linear model. In addition, the $\mathrm{SUV}_{\max }$ ratio of the verified focal lesions were correlated to serum concentrations of insulin, proinsulin and C-peptide, and to the insulin-to-glucose ratio. Corresponding $p$ values were calculated by $t$ tests.

All data analyses were performed using the statistical software program R, version 3.1.2 [37], including several packages [38-44]. Level of significance was $p<0.05$, trends $0.05-0.10$.

\section{Results}

Of the 55 children with $\mathrm{CHI}, 53$ had a PET/CT scan with $18 \mathrm{~F}-\mathrm{DOPA}$, and $18 \mathrm{had}$ a $68 \mathrm{Ga}-\mathrm{DOTANOC}$ scan, of which 16 also had an 18F-DOPA scan (Table 1). The median (IQR) age at PET/CT scan was 7 (3.518.5) months. Among patients who underwent surgery $(n=34)$, pathology reports showed focal CHI in 22 $(64.7 \%)$, diffuse CHI in $10(29.4 \%)$, atypical CHI in one $(2.9 \%)$ and normal pancreatic histology in the analyzed tissue in one $(2.9 \%$; Table 2$)$. The patients with non-focal CHI who underwent surgery were unresponsive to medication with high risk of (further) brain damage from hypoglycaemia. The operated upon patient with normal pancreatic tail histology and three patients without surgery had mutations of uncertain pathogenicity and were excluded from analyses of test performance and ROC.

Examples of 18F-DOPA PET/CT and 68Ga-DOTANOC PET/CT are shown in Fig. 1.

The kappa value for the visual evaluation of the 51 18F-DOPA PET/CT scans was 0.74 (95\% CI 0.55-0.93) and $0.89(0.68-1)$ for the $1868 \mathrm{Ga}-\mathrm{DOTANOC} \mathrm{PET/CT}$. The ICC of the $\mathrm{SUV}_{\max }$ ratio values of the $18 \mathrm{~F}-\mathrm{DOPA}$ PET scans was $0.96(0.94-0.98)$. For 68Ga-DOTANOC PET, the ICC of the $\mathrm{SUV}_{\max }$ values was $0.95(0.88-$ 0.98). Additional ICC values for $68 \mathrm{Ga}-\mathrm{DOTANOC}$ PET are shown in Online Resource Table 1.

\section{Evaluation of 18F-DOPA PET/CT}

Visual evaluation of 18F-DOPA PET/CT showed a sensitivity of $1(0.85-1)$, specificity of $0.96(0.82-0.99)$, a PPV of $0.96(0.79-0.99)$ and an NPV of 1 (0.87-1) for the prediction of focal CHI (Table 3a). Equivalent results were obtained when only histology was used as the isolated gold standard (Table 3b). Tracer uptake in the gall bladder and common bile duct was seen once at the $10-\mathrm{min}$ series and more frequently at 30 or $60 \mathrm{~min}$. The variable staining allowed discrimination of common bile duct staining from suspected focal lesion.

The maximal tracer values by $18 \mathrm{~F}-\mathrm{DOPA}$ PET were obtained at $10 \mathrm{~min}$ in 12 patients (focal $n=4$ ), at $30 \mathrm{~min}$ in 13 patients (focal $n=5$ ) and at $60 \mathrm{~min}$ in 24 patients (focal $n=13$ ). $\mathrm{SUV}_{\max }$ ratio values of the focal lesions had a median (IQR) of 1.72 (1.58-2.27), ranging from 1.47 to 4.69 , compared to 1.12 (1.05$1.20)$, with a range of $0.94-1.73$, for the non-focal type 
Table 1 Patient characteristics

\section{Patients}

Country of origin, no. (\%)

2 (3.6)

Denmark and Greenland

13 (23.6)

Kazakhstan

$3(5.5)$

Latvia

$2(3.6)$

Norway

$1(1.8)$

Russia

15 (27.3)

Sweden

$10(18.2)$

Syria

$1(1.8)$

Ukraine

7 (12.7)

United Kingdom

$1(1.8)$

Sex

Female (\%)

Male (\%)

$30(54.5)$

Age at diagnosis (month), median $(\mathrm{IQR})^{\mathrm{a}}$, no. $=54$

$0(0-0)$

Age at PET/CT (month), median (IQR), no. $=55$

$7(3.5-18.5)$

Surgery, no. (\%)

$34(61.8)$

Disease severity, median (IQR)

Lowest blood glucose $(\mathrm{mmol} / \mathrm{L})$, no. $=55$

$1.0(0.6-1.6)$

Glucose demand ${ }^{\mathrm{b}}(\mathrm{mg} / \mathrm{kg} / \mathrm{min})$, no. $=51$

$8.5(4.4-12.1)$

P-insulin during hypoglycaemia $(\mathrm{pmol} / \mathrm{L})^{\mathrm{d}}$, no. $=37$

$90(48-115)$

Insulin-to-glucose $\mathrm{ratio}^{\mathrm{c}}(\mathrm{pmol} / \mathrm{mmol})$ no. $=37$

$38.4(18.1-55.6)$

Genetic mutations, no. (\%)

ABCC8

Paternal

22

Maternal

Compound heterozygous

1

8

Homozygous

De novo

1

KCNJ11

Paternal

Maternal 3

GLUD1

1

UPD11

HNF4-alfa

No disease-causing mutations found

${ }^{\text {a }}$ Range: $0-9$ months

${ }^{\mathrm{b}}$ Medication-free

${ }^{\mathrm{c}}$ Obtained during hypoglycaemia (glucose $<2.5 \mathrm{mmol} / \mathrm{L}$ for age $<3$ days, glucose $<3.2 \mathrm{mmol} / \mathrm{l}$ for age $\geq 3$ days)

${ }^{\mathrm{d}} \mathrm{P}$-insulin at low p-glucose, reference $<18 \mathrm{pmol} / \mathrm{L}$

$(p<0.0001)$. The classified non-focal patient with an $\mathrm{SUV}_{\max }$ ratio of 1.73 was not subjected to surgery and had a normal $A B C C 8 / K C N J 11$ genetic analysis, which may represent a false negative genotype for focal CHI.

The performance of 18F-DOPA PET was excellent with an ROC AUC of 0.98 (0.93-1; Fig. 2a). The optimal $\mathrm{SUV}_{\max }$ ratio cut-off was 1.44 (1.35-1.46). Equivalent results were obtained when using histology as the isolated gold standard (Fig. 2c).

Quantitative evaluation of 18F-DOPA PET using the cutoff of 1.44 showed the exact same estimates for test performance as the visual evaluation (Table 3). 
Table 2 Gold standard for type of CHI

\begin{tabular}{ll}
\hline & Number $(\%)$ \\
\hline All patients & 55 \\
Included patients & $51(92.7)$ \\
Histology (after surgery): $n=33$ & $22(64.7)$ \\
Focal & \\
Non-focal & $10(29.4)$ \\
$\quad$ Diffuse & $1(2.9)$ \\
Atypical & \\
Genetics (no. surgery): $n=18$ & $0(0)$ \\
Suspected focal & \\
Suspected non-focal & $18(85.7)$ \\
ABCC8: maternal, heterozygous & 1 \\
ABCC8: compound heterozygous & 1 \\
ABCC8: homozygous & 1 \\
GLUD1: heterozygous & 1 \\
HNF4-alfa: heterozygous & 1 \\
No mutations found & 13 \\
Excluded patients & $4(7.3)$ \\
Normal histology report & $1(2.9)$ \\
Genetic variants of uncertain pathogenicity & $3(14.3)$ \\
$A B C C 8:$ paternal & 1 \\
ABCC8: de novo & 1 \\
KCNJ11: maternal & 1 \\
\hline
\end{tabular}

${ }^{a}$ Heterozygous paternal $A B C C 8 / K C N J 11$ mutation

\section{Evaluation of 68Ga-DOTANOC PET/CT}

Visual evaluation of $68 \mathrm{Ga}$-DOTANOC PET/CT showed a test performance with a sensitivity of $0.78(0.45-$ $0.94)$, a specificity of $0.86(0.49-0.97)$, a PPV at 0.88 (0.53-0.98) and an NPV at $0.75(0.41-0.93)$. Equivalent results were obtained when histology was used as the isolated gold standard (Table 3).

The maximal tracer values by $68 \mathrm{Ga}-\mathrm{DOTANOC}$ PET were obtained in 30-60 $\mathrm{min}$ (zero patients at $10 \mathrm{~min}$, two patients at $30 \mathrm{~min}$; focal $n=1$ ), seven patients at $45 \min$ (focal $n=3$ ) and seven patients at $60 \mathrm{~min}$ (focal $n=5$ ). $\mathrm{SUV}_{\max }$ values of the focal lesions had a median (IQR) of $9.43(5.86-12.51)$ with a range of $2.32-16.81 \mathrm{~g} / \mathrm{ml}$, compared to $4.71(3.27-6.98)$ with a range $2.31-13.42 \mathrm{~g} / \mathrm{ml}$ for the non-focal type $(p=0.17)$.

The diagnostic performance of $\mathrm{SUV}_{\max }$ in $68 \mathrm{Ga}$ DOTANOC PET was fair with an ROC AUC of 0.71 (0.430.95; Fig. 2b). The optimal SUV $\max$ cut-off was 6.77 (3.479.90) $\mathrm{g} / \mathrm{ml}$. Test performance showed a sensitivity of 0.67 (0.35-0.88), a specificity of $0.71(0.36-0.92)$, a PPV of 0.75 (0.41-0.93) and an NPV of $0.63(0.31-0.86)$. No significant difference was obtained if applying histology only as the gold standard (Fig. 2d, Table 3).
Differences between point estimates of the visual analysis vs. SUV $\mathrm{max}_{\max }$ cut-offs by $68 \mathrm{Ga}$-DOTANOC PET did not reach significance.

\section{DOPA vs. DOTANOC scans}

The ROC AUC of 18F-DOPA PET showed a superior performance compared to 68Ga-DOTANOC PET; $p=0.025$ (histology and genetic analysis as the gold standard), and $p=0.017$ (histology only as the gold standard).

The 18F-DOPA PET/CT (by visual or SUV ratio) detected the correct location in $91 \%(20 / 22)$ of the cases with focal lesions. Two lesions were incorrectly located. The first patient with an incorrectly located focal lesion (from 2007, no supplementary 68Ga-DOTANOC PET scan and no diagnostic CT scan) was deemed to be located in the head of the pancreas; the lesion was later identified at the tip of the pancreatic tail. The second patient (from 2016, no supplementary 68GaDOTANOC PET scan) had an ectopic lesion in the duodenum near the major duodenal papilla. The lesion was horseshoeshaped and extended into the pancreas. The pancreatic part of the lesion was correctly located by the PET/CT but the true nature of the lesion was classified by histology.

The 68Ga-DOTANOC PET/CT (visual and $\mathrm{SUV}_{\max }$ ) scans detected the correct location in 7/7 and 6/6 of the cases.

\section{Ga-DOTANOC PET SUV max $_{\text {ratios }}$}

In a post-hoc analysis of $68 \mathrm{Ga}$-DOTANOC PET, we attempted calculating $\mathrm{SUV}_{\max }$ ratios by use of normal pancreatic tissue, spleen or liver as reference. The resulting ROC curve and test values were not significantly different from the use of pancreatic $\mathrm{SUV}_{\max }$. Data are given in Online Resource Table 2 and Online Resource Fig. 1.

\section{Disease severity and $S U V_{\max }$ ratio}

No relationship was observed between the minimal blood glucose, or maximal glucose infusion demand, and the $18 \mathrm{~F}-$

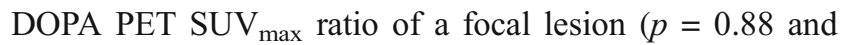
$p=0.39$, respectively; Fig. 3a-b). Equivalent results were obtained when analyzing serum insulin, C-peptide, proinsulin and insulin-to-glucose ratios (Fig. 3c-f).

\section{Discussion}

In this blinded retrospective study, we found significantly better performance of $18 \mathrm{~F}-\mathrm{DOPA} \mathrm{PET} / \mathrm{CT}$ compared to $68 \mathrm{Ga}-$ DOTANOC PET/CT in preoperative prediction of focal CHI. 


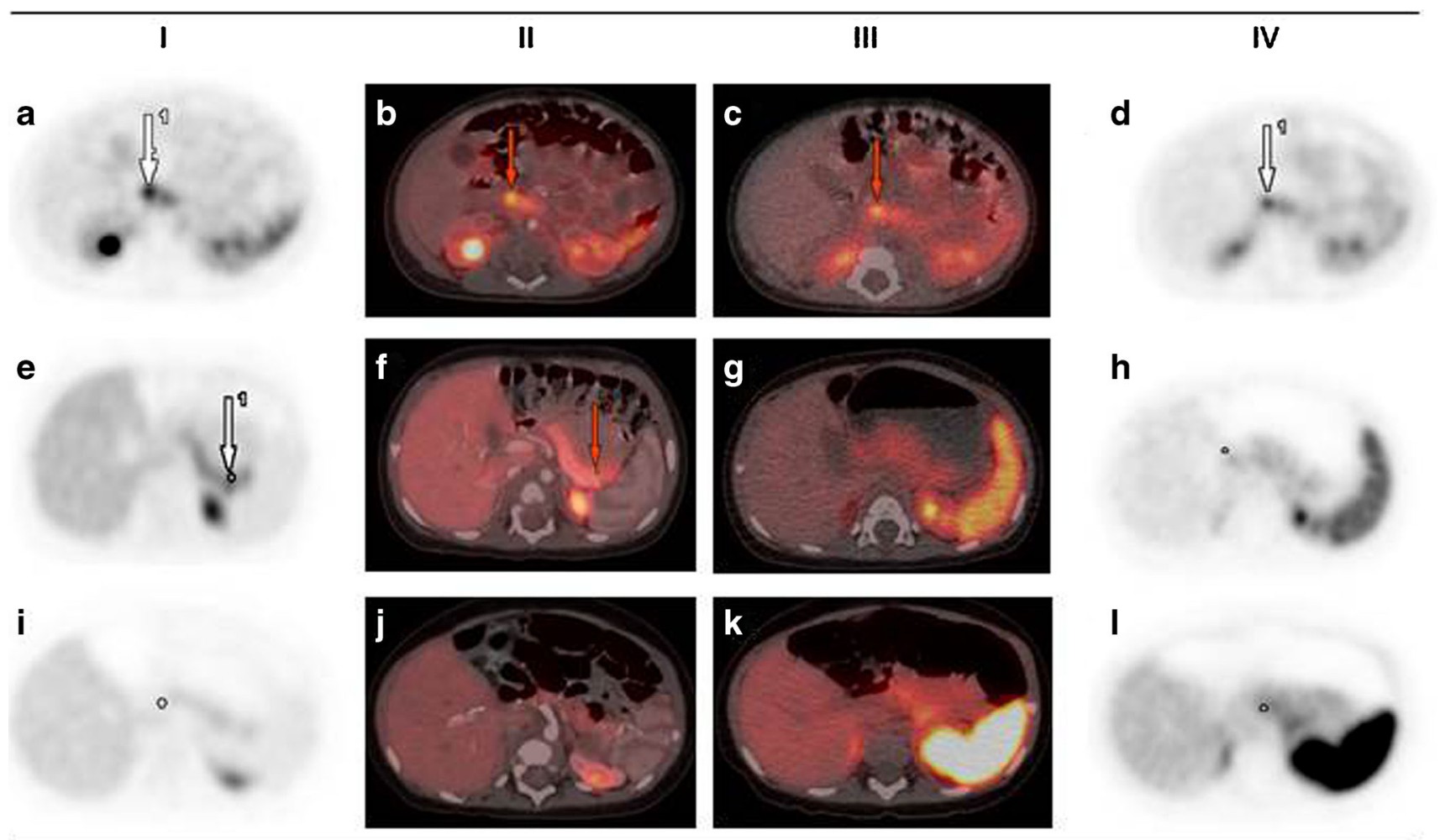

Fig. 1 18F-DOPA PET/CT and 68Ga-DOTANOC PET/CT performed in three patients. a-d Patient 1, focal CHI. e-h Patient 2, focal CHI. i-l Patient 3, diffuse CHI. Column I shows 18F-DOPA PET; column II 18FDOPA PET/CT (here contrast-enhanced); column III 68Ga-DOTANOC $\mathrm{PET} / \mathrm{CT}$; column IV 68Ga-DOTANOC PET. Red arrows point to the

For $18 \mathrm{~F}-\mathrm{DOPA}$ PET/CT, the visual criteria performed identically excellent as compared with an optimal $\mathrm{SUV}_{\max }$ ratio cutoff of 1.44 after ROC curve evaluation. Clinical disease severity did not correlate with the size of the $\mathrm{SUV}_{\max }$ ratio.

Only a few case reports have been published regarding the use of $68 \mathrm{Ga}-D O T A N O C$ in the prediction of focal $\mathrm{CHI}$, and with variable success $[24,45]$. The wider availability of $68 \mathrm{Ga}-$ focal lesions. In the blinded evaluations, patient 1 and 2 were correctly diagnosed as focal and patient 3 as non-focal CHI by $18 \mathrm{~F}-$ DOPA PET/ CT (visual and by cut-off method). By 68Ga-DOTANOC PET/CT, patient 1 and patient 3 were correctly classified, but patient 2 was wrongly classified as non-focal

DOTANOC could argue for the use of this tracer, but the need of a systematic evaluation of the performance of $68 \mathrm{Ga}-$ DOTANOC has not been met until now.

Our optimal $\mathrm{SUV}_{\max }$ ratio cut-off in $18 \mathrm{~F}-\mathrm{DOPA}$ PET to predict focal CHI was 1.44. To the best of our knowledge, this is the first attempt to classify an $\mathrm{SUV}_{\max }$ ratio cut-off in CHI by the use of the ROC. Others have advocated a cut-off ratio

Table 3 Test performance of 18F-DOPA PET/CT and 68GA-DOTANOC PET/CT in predicting focal CHI

\begin{tabular}{lclllll}
\hline Method & No. & Sensitivity $(95 \%$ CI $)$ & Specificity (95\% CI) & PPV (95\% CI) & NPV (95\% CI) & Location ${ }^{\text {a }(\%)}$ \\
\hline a) Patients with histology or genetics as gold standard & 49 & $1(0.85-1)$ & $0.96(0.82-0.99)$ & $0.96(0.79-0.99)$ & $1(0.87-1)$ \\
18F-DOPA PET visual & 49 & $1(0.85-1)$ & $0.96(0.82-0.99)$ & $0.96(0.79-0.99)$ & $1(0.87-1)$ & $20 / 22(91)$ \\
18F-DOPA PET cut-off 1.44 & 16 & $0.78(0.45-0.94)$ & $0.86(0.49-0.97)$ & $0.88(0.53-0.98)$ & $0.75(0.41-0.93)$ & $7 / 7(100)$ \\
68Ga-DOTANOC PET visual & 16 & $0.67(0.35-0.88)$ & $0.71(0.36-0.92)$ & $0.75(0.41-0.93)$ & $0.63(0.31-0.86)$ & $6 / 6(100)$ \\
68Ga-DOTANOC PET cut-off 6.77 & & & & & & \\
b) Patients with histology as gold standard & & $1(0.72-1)$ & $1(0.85-1)$ & $1(0.72-1)$ & $20 / 22(91)$ \\
18F-DOPA PET visual & 32 & $1(0.85-1)$ & $1(0.72-1)$ & $1(0.85-1)$ & $1(0.72-1)$ & $20 / 22(91)$ \\
18F-DOPA PET cut-off 1.44 & 32 & $1(0.85-1)$ & $0.80(0.38-0.96)$ & $0.88(0.53-0.98)$ & $0.67(0.30-0.90)$ & $7 / 7(100)$ \\
68Ga-DOTANOC PET visual & 14 & $0.78(0.45-0.94)$ & $0.60(0.23-0.88)$ & $0.71(0.36-0.92)$ & $0.43(0.16-0.75)$ & $5 / 5(100)$ \\
68Ga-DOTANOC PET cut-off 7.73 & 14 & $0.56(0.27-0.81)$ & & & &
\end{tabular}

${ }^{\text {a }}$ Correctly located/correct focal identification according to histology after surgery

PPV, positive predictive value, NPV, negative predictive value 

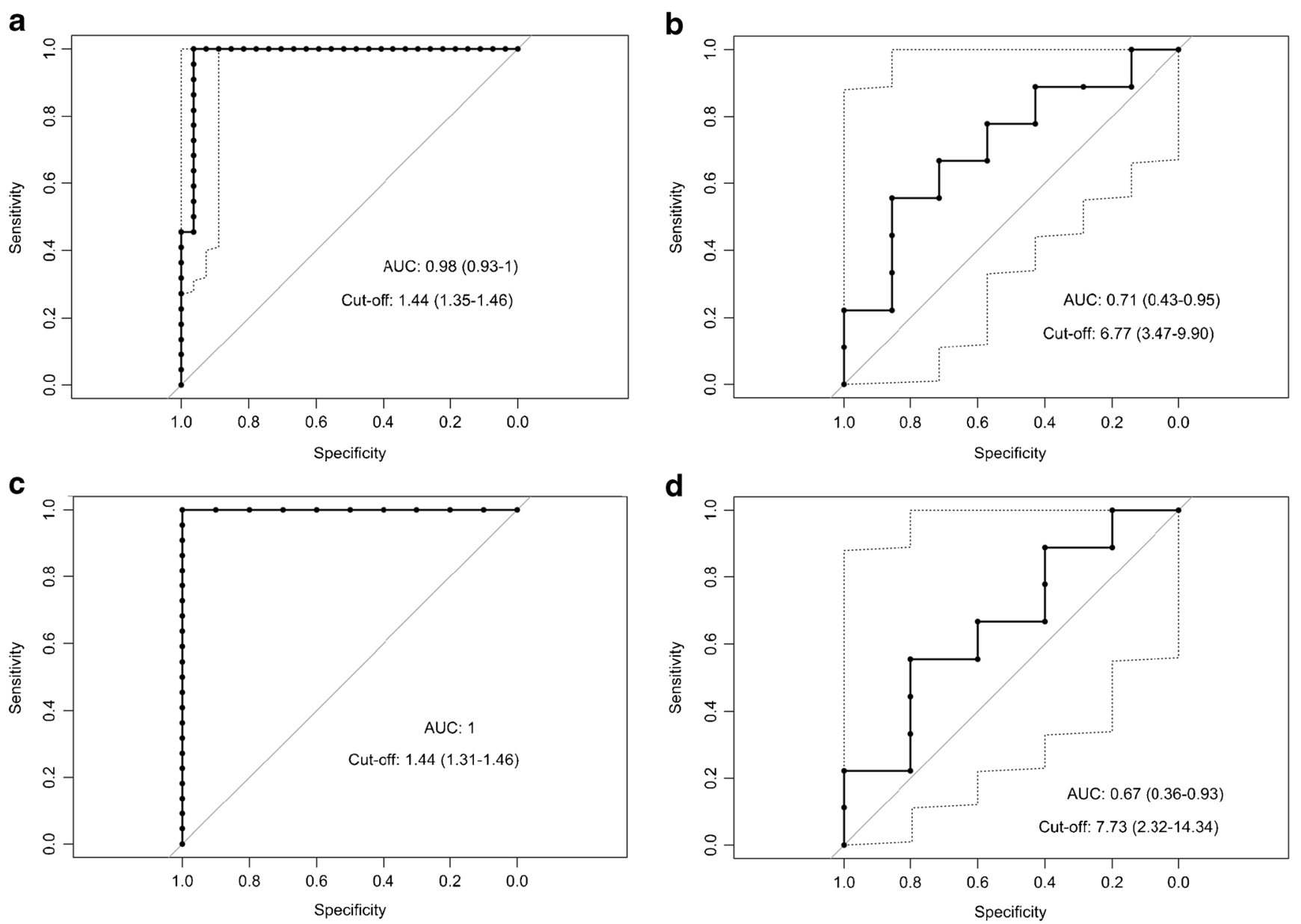

Fig. 2 ROC curves for the performance of PET/CT scans in predicting focal CHI. a 18F-DOPA PET, SUV ratio, b 68Ga-DOTANOC PET, SUV max, $\mathbf{c}$ 18F-DOPA PET, SUV ratio with histology as the singular gold standard, $\mathbf{d} 68 \mathrm{Ga}-\mathrm{DOTANOC}$ PET, SUV $_{\text {max }}$ with histology as the singular gold standard

between 1.2 [22] and $1.5[17,19,20]$, either with SUV nominator and denominator values measured as mean SUV $\left(\mathrm{SUV}_{\text {mean }}\right)[17,22]$, or as $\operatorname{SUV}_{\max }[19,20]$.

Ribeiro et al. [21] calculated the SUV ratio using the $\mathrm{SUV}_{\text {mean }}$ of the ROI divided by the $\mathrm{SUV}_{\text {mean }}$ of the pancreas. They found an average (range) SUV ratio of $1.44(1.2-1.8)$ in 14 patients with focal CHI. The average value of 1.44 was exactly the same as our optimal cut-off, but far below our

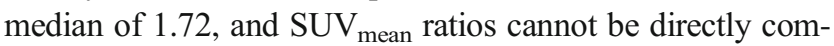
pared to $\mathrm{SUV}_{\text {max }}$ ratios.

To the best of our knowledge, the current study is the first blinded comparison of the visual judgment vs. an SUV ratio cut-off in CHI patients. The test performance of the $18 \mathrm{~F}-$ DOPA PET/CT was identical when analyzed visually and when the optimal $\mathrm{SUV}_{\max }$ ratio cut-off was applied. Others have advocated for a visual judgment $[15,18,21]$, but only one study reported a blinded evaluation [15]. In practice, a combination of all available data may be helpful in the interpretation of PET/CT scan results, but this will bias the assessment and the diagnostic tool performance. The blinded $\mathrm{SUV}_{\max }$ ratio cut-off 1.44 should be validated in another population with the same methodology [46]. Our test performance results of 18F-DOPA PET were in line with the results of a meta-analysis [47], which found a pooled AUC of 0.95 , a sensitivity of $0.89(0.81-0.95)$ and a specificity of 0.98 (0.89-1). The meta-analysis did not differ between visual or quantitative analysis of the PET scans or the method to obtain SUV ratios. Kühnen et al. have in a more recent, unblinded study found a sensitivity for predicting focal CHI of $100 \%$ [48]. However, in 3 of their 32 patients, the extent of giant focal lesions was severely underestimated by $18 \mathrm{~F}-\mathrm{DOPA}$ PET. In our series, no giant focal lesions were present, which is why the performance of $18 \mathrm{~F}-\mathrm{DOPA}$ PET $v s$. $68 \mathrm{Ga}-$ DOTANOC PET could not be estimated for such lesions.

The combination of 18F-DOPA PET and CT (or MRI) is an advantage for obtaining superimposed pictures of both modalities as guidance for the surgeons. We, as others [17, 19], stress the use of diagnostic CT when a focal lesion is suspected. In fact, one of our two failures in predicting the location of the focal $\mathrm{CHI}$ lesion occurred in one of our first patients, in whom diagnostic $\mathrm{CT}$ as an exception was not performed, which is why a tail focus was impossible to discern from kidney uptake. The other blinded localization failure by 18F-DOPA PET/CT correctly located the lesion in the 

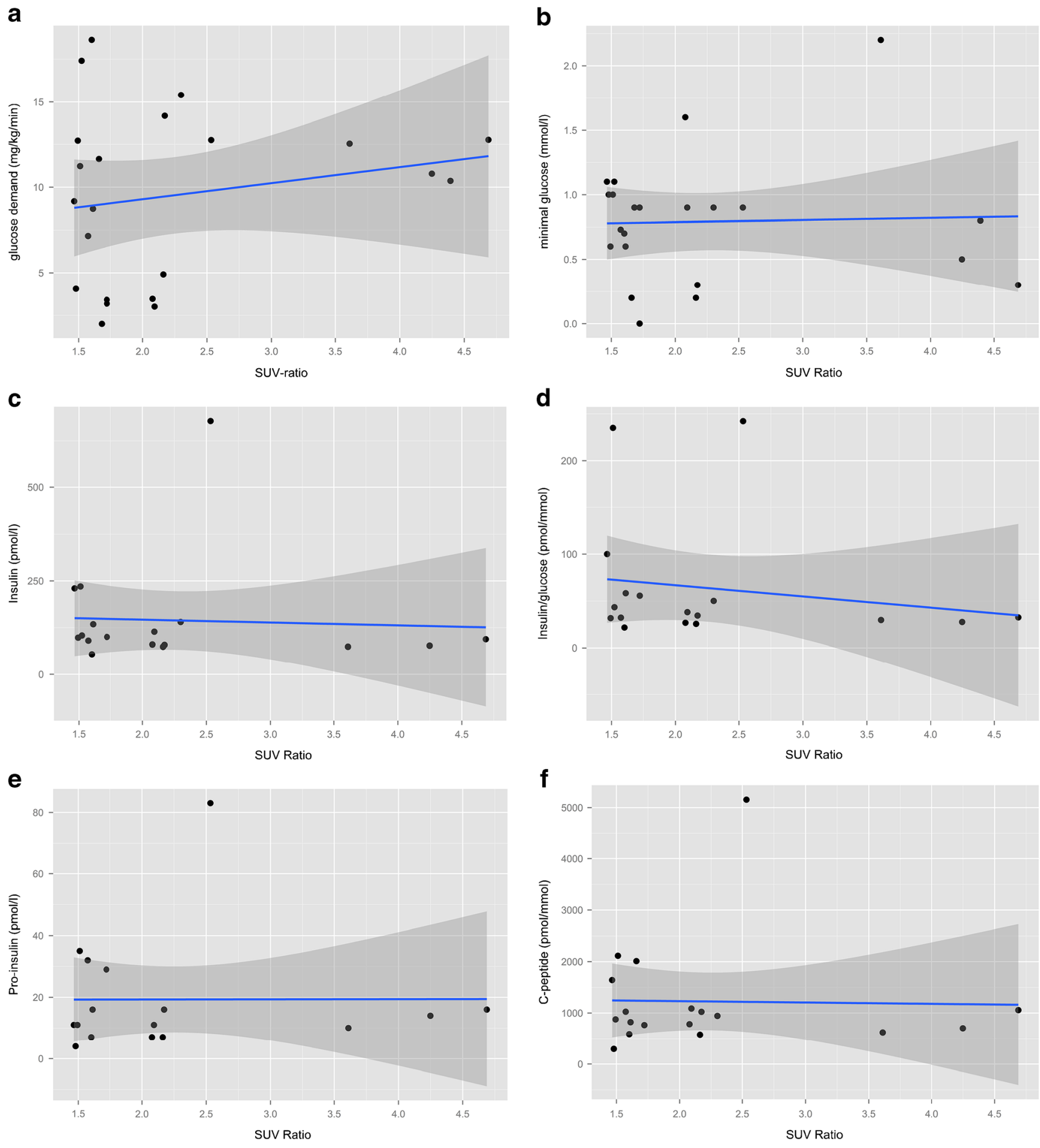

Fig. 3 Correlation of focal SUV ratio and disease severity. a Intravenous glucose demand, b minimal glucose value, $\mathbf{c}$ insulin concentration (pmol/L), d insulin-to-glucose ratio ( $\mathrm{pmol} / \mathrm{L}: \mathrm{mmol} / \mathrm{L})$, pro-insulin

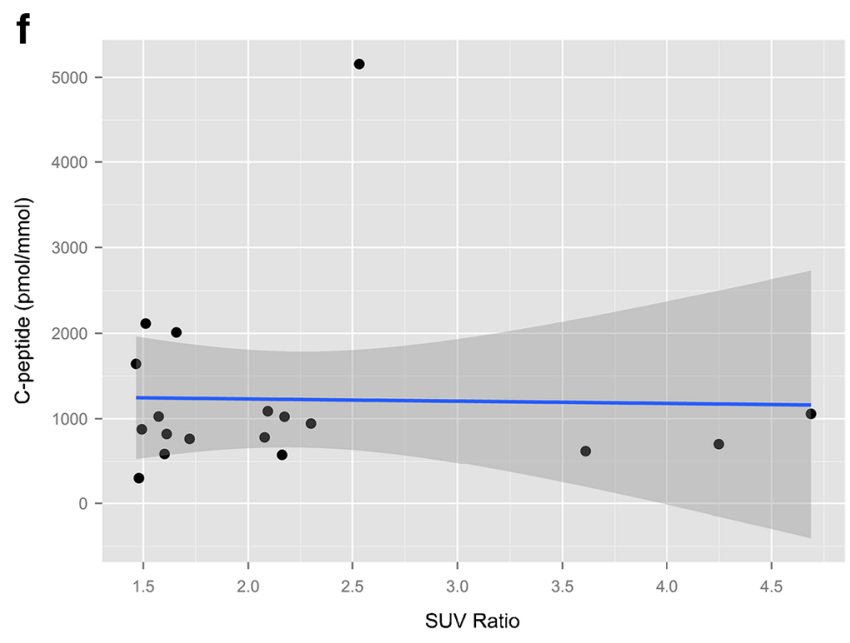

concentration (pmol/L) and e C-peptide concentration (pmol/L). The gray area indicates the $95 \% \mathrm{CI}$ for the linear model

pancreatic head, but failed to identify that the lesion was in fact ectopic and situated in the duodenal wall, extending into the pancreas. In fact, the possibility of an ectopic lesion was raised in the nuclear medicine report, as seen after unblinding. Focal ectopic lesions are rare and this has led to extensive

redundant pancreatic resections before the introduction of 18F-DOPA PET/CT [49, 50]. For 68Ga-DOTANOC PET/ $\mathrm{CT}$, caution should be raised to not interpret labeling in an accessory spleen as an ectopic or pancreatic tail focal lesion, as $68 \mathrm{Ga}$-DOTANOC PET shows physiological labeling in 
splenic tissue in contrast to $18 \mathrm{~F}-\mathrm{DOPA}$ PET. No such errors were seen in our blinded study, nor in the clinical setting.

We took the opportunity to evaluate the glucose demand and the hyperinsulinism diagnosis during a short hypoglycaemia after discontinuation of medication. This procedure allowed us to reveal that the glucose infusion rate offmedication, as well as other biochemical parameters, did not correlate with the size of the $\mathrm{SUV}_{\text {max }}$ ratio in focal CHI. This is in keeping with a case report of a child with a high $\mathrm{SUV}_{\max }$ (no ratio given) and a visually clear focal lesion both before and after clinical remission [51].

Not all CHI centers use time series [20-22] or general anesthesia $[19,21,22]$ for $18 \mathrm{~F}-\mathrm{DOPA}$ PET/CT as we did. Our time series protocol revealed that the time of maximal SUV varied from 10 to $60 \mathrm{~min}$ in $18 \mathrm{~F}-\mathrm{DOPA}$ and 30 to $60 \mathrm{~min}$ in $68 \mathrm{Ga}$-DOTANOC scans, suggesting the advantage of a time series at least in $18 \mathrm{~F}-\mathrm{DOPA}$ PET, where intrapancreatic bile duct tracing may occur. General anesthesia was preferred in most of our patients with prolonged need for an unmoving child.

\section{Strengths and limitations}

Strengths of our study included the blinded analysis by two researchers with different experiences and a third blinded researcher to decide any disagreements. The high kappa and ICC values showed that the PET/CT scan estimates were robust and that knowledge of genetic and clinical details was not mandatory for an excellent diagnostic performance. Moreover, we used $\mathrm{SUV}_{\max }$, which is more reproducible than $\mathrm{SUV}_{\text {mean }}[52]$, and bias from a single or very few pixels was minimized by use of an ROI shaped as a sphere. Lastly, we used both histology and genetics as gold standards, but calculations for the histology gold standard alone did not change the estimates.

Limitations included the retrospective nature of the study, potential misclassification of focal vs. diffuse $\mathrm{CHI}$ based on genetic results in patients not subjected to surgery, and the earliest used dHPLC method in the genetic analysis, believed to be of lower quality [53].

\section{Conclusion}

In conclusion, blinded 18F-DOPA PET/CT interpretation was excellent in predicting focal $\mathrm{CHI}$ and localizing the lesion. $68 \mathrm{Ga}-\mathrm{DOTANOC}$ PET had a lower point estimate of the ROC AUC and superiority of 18F-DOPA PET/CT was seen, which is why further use of 68GA-DOTANOC PET/CT in predicting focal $\mathrm{CHI}$ is discouraged.

\section{Ethics}

The study was approved by the Danish Health Research Ethics Committee (protocol no. 48572), and the Danish Data Protection Authority (protocol no. 2015-41-3867). For this type of study, formal consent was not required.

Acknowledgements We thank MD Anders Thomassen and MD Mie Holm Vilstrup for help with blinded PET scan analyses and cand. scient. oecon., Ph.D. Oke Gerke and M.D. Marc T. K. Nielsen for statistical help. A special thanks to the patients, their parents and all the referring hospitals. Furthermore, we thank Miss Olha Volkova, the Danish-Ukraine Friendship Organization and Lene Boesen at Hans Christian Andersen Children's Hospital, Odense University Hospital, Odense, Denmark.

Source of Funding This project was funded by the Danish Council for Independent Research.

\section{References}

1. Arnoux J-B, Verkarre V, Saint-Martin C, Montravers F, Brassier A, Valayannopoulos $\mathrm{V}$, et al. Congenital hyperinsulinism: current trends in diagnosis and therapy. Orphanet J Rare Dis. 2011;6:63.

2. Otonkoski T, Ammälä C, Huopio H, Cote GJ, Chapman J, Cosgrove $\mathrm{K}$, et al. A point mutation inactivating the sulfonylurea receptor causes the severe form of persistent hyperinsulinemic hypoglycemia of infancy in Finland. Diabetes. 1999;48:408-15.

3. Yorifuji T, Masue M, Nishibori H. Congenital hyperinsulinism: global and Japanese perspectives. Pediatr Int. 2014;56:467-76.

4. Helleskov A, Melikyan M, Globa E, Shcherderkina I, Poertner F, Larsen A-M, et al. Both low blood glucose and insufficient treatment confer risk of neurodevelopmental impairment in congenital hyperinsulinism: a multinational cohort study. Front Endocrinol. 2017;8:156. https://doi.org/10.3389/fendo.2017.00156. eCollection 2017

5. Lord K, Dzata E, Snider KE, Gallagher PR, De León DD. Clinical presentation and Management of Children with Diffuse and Focal Hyperinsulinism: a review of 223 cases. J Clin Endocrinol Metabol. 2013;98:E1786-9.

6. Snider KE, Becker S, Boyajian L, Shyng S-L, MacMullen C, Hughes N, et al. Genotype and phenotype correlations in 417 children with congenital Hyperinsulinism. J Clin Endocrinol Metab. 2013;98:E355-63.

7. Rahier J, Guiot Y, Sempoux C. Morphologic analysis of focal and diffuse forms of congenital hyperinsulinism. Semin Pediatr Surg. 2011;20:3-12.

8. Kapoor RR, Flanagan SE, Arya VB, Shield JP, Ellard S, Hussain K. Clinical and molecular characterisation of 300 patients with congenital hyperinsulinism. Eur J Endocrinol. 2013;168:557-64.

9. Shah P, Demirbilek H, Hussain K. Persistent hyperinsulinaemic hypoglycaemia in infancy. Semin Pediatr Surg. 2014;23:76-82.

10. Hussain K, Blankenstein O, De Lonlay P, Christesen HT. Hyperinsulinaemic hypoglycaemia: biochemical basis and the importance of maintaining normoglycaemia during management. Arch Dis Child. 2007;92:568-70.

11. Beltrand J, Caquard M, Arnoux J-B, Laborde K, Velho G, Verkarre $\mathrm{V}$, et al. Glucose metabolism in 105 children and adolescents after pancreatectomy for congenital Hyperinsulinism. Diabetes Care. 2012;35:198-203.

12. Arya VB, Senniappan S, Demirbilek H, Alam S, Flanagan SE, Ellard S, et al. Pancreatic endocrine and exocrine function in 
children following near-total pancreatectomy for diffuse congenital hyperinsulinism. PLoS One. 2014;9:e98054.

13. Blomberg BA, Moghbel MC, Saboury B, Stanley CA, Alavi A. The value of radiologic interventions and $18 \mathrm{~F}-\mathrm{DOPA}$ PET in diagnosing and localizing focal congenital Hyperinsulinism: systematic review and meta-analysis. Mol Imaging Biol. 2012;15:97-105.

14. de Lonlay P, Simon-Carre A, Ribeiro M-J, Boddaert N, Giurgea I, Laborde $\mathrm{K}$, et al. Congenital hyperinsulinism: pancreatic [18F]fluoro-L-dihydroxyphenylalanine (DOPA) positron emission tomography and immunohistochemistry study of DOPA decarboxylase and insulin secretion. J Clin Endocrinol Metab. 2006;91:933-40.

15. Laje P, States LJ, Zhuang H, Becker SA, Palladino AA, Stanley CA, et al. Accuracy of PET/CT scan in the diagnosis of the focal form of congenital hyperinsulinism. J Pediatr Surg. 2013;48:388-93.

16. Zani A, Nah SA, Ron O, Totonelli G, Ismail D, Smith VV, et al. The predictive value of preoperative fluorine-18-1-3,4dihydroxyphenylalanine positron emission tomography-computed tomography scans in children with congenital hyperinsulinism of infancy. J Pediatr Surg. 2011;46:204-8.

17. Barthlen W, Blankenstein O, Mau H, Koch M, Höhne C, Mohnike W, et al. Evaluation of [18F]Fluoro-1-DOPA positron emission tomography-computed tomography for surgery in focal congenital Hyperinsulinism. J Clin Endocrinol Metabol. 2008;93:869-75.

18. Hardy OT, Hernandez-Pampaloni M, Saffer JR, Scheuermann JS, Ernst LM, Freifelder R, et al. Accuracy of [18F]Fluorodopa positron emission tomography for diagnosing and localizing focal congenital Hyperinsulinism. J Clin Endocrinol Metabol. 2007;92: 4706-11.

19. Meintjes M, Endozo R, Dickson J, Erlandsson K, Hussain K, Townsend C, et al. 18F-DOPA PET and enhanced CT imaging for congenital hyperinsulinism: initial UK experience from a technologist's perspective. Nucl Med Commun. 2013;34:601-8.

20. Otonkoski T, Näntö-Salonen K, Seppänen M, Veijola R, Huopio H, Hussain K, et al. Noninvasive diagnosis of focal Hyperinsulinism of infancy with [18F]-DOPA positron emission tomography. Diabetes. 2006;55:13-8.

21. Ribeiro M-J, Boddaert N, Bellanné-Chantelot C, Bourgeois S, Valayannopoulos V, Delzescaux T, et al. The added value of [18F]fluoro- L-DOPA PET in the diagnosis of hyperinsulinism of infancy: a retrospective study involving 49 children. Eur J Nucl Med Mol Imaging. 2007;34:2120-8.

22. Capito C, Khen-Dunlop N, Ribeiro M-J, Brunelle F, Aigrain Y, Crétolle C, et al. Value of 18F-fluoro-l-dopa PET in the preoperative localization of focal lesions in congenital Hyperinsulinism. Radiology. 2009;253:216-22.

23. Kumar U, Sasi R, Suresh S, Patel A, Thangaraju M, Metrakos P, et al. Subtype-selective expression of the five somatostatin receptors (hSSTR1-5) in human pancreatic islet cells: a quantitative double-label immunohistochemical analysis. Diabetes. 1999;48: 77-85.

24. Yadav D, Dhingra B, Kumar S, Kumar V, Dutta AK. Persistent hyperinsulinemic hypoglycemia of infancy. J Pediatr Endocrinol Metab. 2012;25:591-3.

25. Tørring PM, Brusgaard K, Ousager LB, Andersen PE, Kjeldsen AD. National mutation study among Danish patients with hereditary haemorrhagic telangiectasia. Clin Genet. 2014;86:123-33.

26. Christesen HBT, Brusgaard K, Alm J, Sjöblad S, Hussain K, Fenger $\mathrm{C}$, et al. Rapid genetic analysis in congenital Hyperinsulinism. Horm Res. 2007;67:184-8.

27. Henningsen MK, Jelsig AM, Andersen H, Brusgaard K, Ousager LB, Hertz JM. Noonan syndrome can be diagnosed clinically and through molecular genetic analyses. Ugeskr Laeg. 2015;177: V12140755.
28. Adzhubei IA, Schmidt S, Peshkin L, Ramensky VE, Gerasimova A, Bork $\mathrm{P}$, et al. A method and server for predicting damaging missense mutations. Nat Methods. 2010;7:248-9.

29. Schwarz JM, Rödelsperger C, Schuelke M, Seelow D. MutationTaster evaluates disease-causing potential of sequence alterations. Nat Methods. 2010;7:575-6.

30. Ng PC, Henikoff S. SIFT: predicting amino acid changes that affect protein function. Nucleic Acids Res. 2003;31:3812-4.

31. Desmet F-O, Hamroun D, Lalande M, Collod-Beroud G, Claustres $\mathrm{M}$, Beroud C. Human splicing finder: an online bioinformatics tool to predict splicing signals. Nucleic Acids Res. 2009;37:e67.

32. Eng L, Coutinho G, Nahas S, Yeo G, Tanouye R, Babaei M, et al. Nonclassical splicing mutations in the coding and noncoding regions of the ATM gene: maximum entropy estimates of splice junction strengths. Hum Mutat. 2004;23:67-76.

33. Reese MG, Eeckman FH, Kulp D, Haussler D. Improved splice site detection in genie. J Comput Biol. 1997;4:311-23.

34. Pertea M, Lin X, Salzberg SL. GeneSplicer: a new computational method for splice site prediction. Nucleic Acids Res. 2001;29: 1185-90.

35. Altman DG, Gardner MJ. Statistics with confidence confidence intervals and statistical guidelines. [Great Britain]: BMJ Books; 2000.

36. Chan IS, Zhang Z. Test-based exact confidence intervals for the difference of two binomial proportions. Biometrics. 1999;55: $1202-9$.

37. R Development Core Team. R: A Language and Environment for Statistical Computing [Internet]. Vienna, Austria: R Foundation for Statistical Computin; 2008. Available from: http://www.R-project.org.

38. Wickham H. Ggplot2: elegant graphics for data analysis. New York: Springer; 2009.

39. Robin X, Turck N, Hainard A, Tiberti N, Lisacek F, Sanchez J-C, et al. pROC: an open-source package for R and S+ to analyze and compare ROC curves. BMC Bioinforma. 2011;12:77.

40. Wickham H, Francois R. dplyr: A Grammar of Data Manipulation [Internet]. 2016. Available from: http://CRAN.R-project.org/ package $=$ dplyr.

41. Wickham H. The split-apply-combine strategy for data analysis. J Stat Softw. 2015;40:1-29.

42. Dorai-Raj S. Binom: Binomial Confidence Intervals For Several Parameterizations [Internet]. 2014. Available from: http://CRAN. R-project.org/package=binom.

43. Revelle W. psych: Procedures for Psychological, Psychometric, and Personality Research [Internet]. Evanston, Illinois: Northwestern University; 2015. Available from: http://CRAN.R-project.org/ package $=$ psych.

44. Gamer M, Lemon J, PuspendraSingh IF. irr: Various Coefficients of Interrater Reliability and Agreement [Internet]. 2012 [cited 2015 Jul 1]. Available from: http://CRAN.R-project.org/package=irr.

45. Dutta S, Venkataseshan S, Bal C, Rao KLN, Gupta K, Bhattacharya A, et al. Novel use of somatostatin receptor scintigraphy in localization of focal congenital Hyperinsulinism: promising but fallible. J Pediatr Endocrinol Metab. 2009;22:965-70.

46. Thie JA. Understanding the standardized uptake value, its methods, and implications for usage. J Nucl Med. 2004;45:1431-4.

47. Treglia G, Mirk P, Giordano A, Rufini V. Diagnostic performance of fluorine-18-dihydroxyphenylalanine positron emission tomography in diagnosing and localizing the focal form of congenital hyperinsulinism: a meta-analysis. Pediatr Radiol. 2012;42:1372-9.

48. Kühnen P, Matthae R, Arya V, Hauptmann K, Rothe K, Wächter S, et al. Occurrence of giant focal forms of congenital hyperinsulinism with incorrect visualization by $18 \mathrm{~F}$ DOPA-PET/CT scanning. Clin Endocrinol. 2014;81:847-54.

49. Peranteau WH, Mehdi Bathaii S, Pawel B, Hardy O, Alavi A, Stanley CA, et al. Multiple ectopic lesions of focal islet 
adenomatosis identified by positron emission tomography scan in an infant with congenital hyperinsulinism. J Pediatr Surg. 2007;42: 188-92.

50. Hussain K, Seppänen M, Näntö-Salonen K, Adzick NS, Stanley CA, Thornton P, et al. The diagnosis of ectopic focal Hyperinsulinism of infancy with [18F]-Dopa positron emission tomography. J Clin Endocrinol Metabol. 2006;91:2839-42.

51. Yorifuji T, Hosokawa Y, Fujimaru R, Kawakita R, Doi H, Matsumoto T, et al. Lasting 18F-DOPA PET uptake after clinical remission of the focal form of congenital hyperinsulinism. Horm Res Paediatr. 2011;76:286-90.

52. Kinahan PE, Fletcher JW. PET/CT standardized uptake values (SUVs) in clinical practice and assessing response to therapy. Semin Ultrasound CT MR. 2010;31:496-505.

53. Christesen HT, Brusgaard K, Hussain K. Recurrent spontaneous hypoglycaemia causes loss of neurogenic and neuroglycopaenic signs in infants with congenital hyperinsulinism. Clin Endocrinol. 2012;76:548-54. 